\title{
The inflammasome: A remote control for metabolic syndrome
}

\author{
Mohamed Lamkanfi ${ }^{1,2}$, Thirumala-Devi Kanneganti ${ }^{3}$ \\ ${ }^{I}$ Department of Biochemistry, Ghent University, Ghent, Belgium; ${ }^{2}$ Department of Medical Protein Research, VIB, Ghent, Belgium; \\ ${ }^{3}$ Department of Immunology, St. Jude Children's Research Hospital, Memphis, TN 38105, USA \\ Cell Research (2012) 22:1095-1098. doi:10.1038/cr.2012.55; published online 10 April 2012
}

Humans can be divided into roughly three groups with distinct microbial communities in their gastro-intestinal tract. The microbiota contributes to metabolic activity in the gastrointestinal tract of the host, but what mechanisms shape the composition of the gut microbiota, and how does a person's 'enterotype' affect metabolic processes in distant organs? Flavell and colleagues shed light on these questions by revealing an important role for inflammasomes in modulating the prevalence of colitogenic species, and by demonstrating that dysbiosis influences susceptibility to non-alcoholic steatohepatitis (NASH) and other manifestations of metabolic syndrome.

The human gastro-intestinal tract is home to a vast community of microbial species that contribute to food digestion and protection against invading pathogens [1]. Recent work showed that three distinct types of microbial communities ('enterotypes') can be distinguished in humans depending on whether bacterial species of the Bacteroides, Prevotella or Ruminococcus genus prevail [2]. Notably, the three enterotypes were linked to differential production of vitamins

Correspondence: Thirumala-Devi Kanneganti Tel: +1-901-595-3634; Fax: +1-901-595-5766 E-mail: Thirumala-Devi.Kanneganti@StJude. org and differences in Body Mass Index [2]. These findings suggest a link between a person's microbiota and susceptibility to obesity, but does the intestinal microbiome also influence manifestations of metabolic syndrome in distant organs such as the liver and the pancreas as well? Recent work with inflammasomedeficient mice provided important cues suggesting this indeed to be the case. Inflammasomes are cytosolic multiprotein complexes of which the clinical importance in autoimmune, infectious and metabolic diseases is increasingly being recognized [3]. These stress- and infection-sensors contain members of the NOD-like receptor (NLR) and HIN200 families that activate the cysteine protease caspase- 1 through the adaptor protein ASC [3, 4]. Inflammasome-activated caspase-1 responds to microbial infections and (sterile) stress conditions by proteolytically maturing the proinflammatory cytokines interleukin (IL)-1 $\beta$ and IL-18, and by inducing pyroptotic cell death [5].

Notably, the gastro-intestinal tracts of mice lacking the essential inflammasome components Nlrp6, Nlrp3, ASC and caspase- 1 were recently found to contain a microbiome profile that was skewed towards colitogenic species of the Prevotellaceae family and the candidate phylum TM7 [6]. Concurrently, mice lacking these inflammasome proteins were hypersusceptible to dextran sodium sulphate (DSS)-induced colitis [6-10]. In agreement with a critical role for the microbiome in regulating colitis susceptibility in these mice, antibiotic treatment rescued the colitic phenotype $[6,9]$. Intriguingly, the colitogenic properties of inflammasome-deficient mice were readily transferred to wild-type mice upon co-housing or co-fostering [6]. A pressing question that remains to be fully addressed is how inflammasomes regulate the colonic microbiome composition. Il-18-deficient mice also presented with an altered fecal microbiota, suggesting this cytokine to be critically involved downstream of inflammasomes in tempering the abundance of colitogenic microbes in the gastro-intestinal tract [6]. One possibility is that IL-18 may regulate processes involved in maintaining a niche in the intestinal tract that is favorable to non-colitogenic bacteria by preferentially clearing colitogenic species. Inflammasome-produced IL-18 may accomplish this, for instance, by regulating the levels of certain defensins, short peptides with bactericidal properties. In this regard, crypts of $\mathrm{Nlrp}^{-/-}$mice were shown to contain altered defensin levels [11]. However, further analysis is required to establish the role of IL-18 in this process, and to dissect the roles of defensins in shaping the microbiome.

Intriguingly, the role of the intestinal microbiota is not limited to 
regulating inflammation in the gastrointestinal tract given that dysbiosis in inflammasome-deficient mice also influenced susceptibility to non-alcoholic steatohepatitis (NASH), obesity and other manifestations of metabolic syndrome [12]. This is illustrated by the observation that antibiotic treatment significantly reduced NASH severity in $\mathrm{ASC}^{-/-}$mice that were fed a methioninecholine-deficient diet (MCDD), and prevented transmission of the phenotype to co-housed wild-type mice [12]. These findings were confirmed in leptin receptor-deficient $(d b / d b)$ mice. The latter develop non-alcoholic fatty liver disease (NAFLD), but this does not spontaneously progress into NASH [13]. Nevertheless, $d b / d b$ mice co-housed with
$A S C^{-/-}$mice presented with increased liver damage and hepatic inflammation compared to single-housed $d b / d b$ mice [12]. In addition, co-housed $d b / d b$ mice gained significantly more weight than single-housed controls, suggesting that inflammasome-associated colitogenic bacteria may contribute to obesity and other manifestations of metabolic syndrome. Indeed, $A S C^{-/-}$mice and cohoused wild-type mice that were fed a high-fat diet gained body weight faster and featured increased hepatic steatosis when compared to singly-housed wildtype controls [12]. Antibiotic treatment returned body weight gain, glucose intolerance and fasting plasma insulin levels in $A S C^{-1-}$ mice to normal levels, confirming a role for the microbiota in altering these metabolic parameters.

To characterize the mechanisms by which inflammasomes contribute to metabolic syndrome, Flavell and colleagues first established that MCDD-fed $\mathrm{Nlrp}^{-/-}$and caspase- $1^{-/-}$mice developed NASH, similar to $A S C^{-/-}$mice [12]. Interestingly, NASH progression was not tempered in $A S C^{-/-}$mice devoid of T and $\mathrm{B}$ cells $\left(\mathrm{ASC}^{--} ; \mathrm{Rag}^{-/-}\right)$, suggesting that inflammasomes drive liver damage and hepatic steatosis mainly through innate immune pathways. Together with the observation that bone marrow chimeric mice specifically lacking ASC or Nlrp3 in hematopoietic cells failed to present with increased disease progression, this suggested inflammasome activation in non-hematopoietic cells to

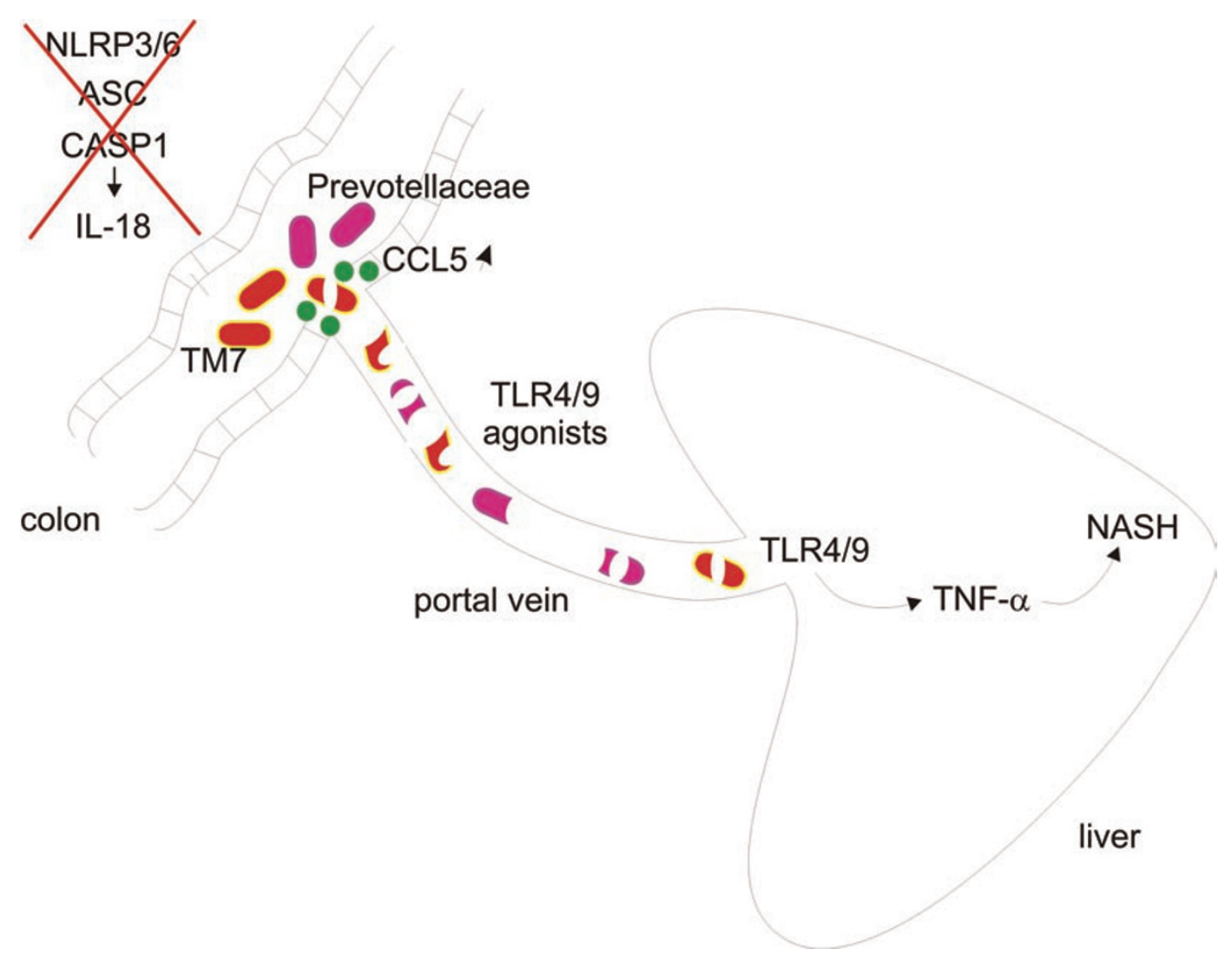

Figure 1 Model for non-alcoholic steatohepatitisprogression in inflammasome-deficient mice. Unlike in wild-type animals, defective IL-18 production in inflammasome-deficient $\mathrm{NIrp6}^{-/-}, \mathrm{NIrp}^{-{ }^{--}}, \mathrm{ASC}^{-/-}$and caspase- $1^{-1-}$ mice skews the intestinal microbiome to colitogenic species characterized by an expanded representation of bacteria from the Prevotellaceae family and the candidate phylum TM7. This increases CCL5 production by the intestinal epithelium, which in turn causes increased intestinal inflammation. Augmented intestinal barrier destruction allows excessive translocation of TLR4 and TLR9 agonists to the liver, where TLR-induced TNF-a production drives hepatic steatosis and inflammation. 
be responsible for NASH exacerbation [12]. The gastro-intestinal tract emerged as a prime location from which inflammasomes regulate metabolic syndrome, as constitutive activation of the Nlrp3 activation in hepatocytes failed to alter NASH progression [12]. Although the protective effect of antibiotic treatment supports this notion, tissue-specific deletion of the Nlrp3 inflammasome in Paneth cells and other intestinal cell types might allow further dissection. Regardless, IL-18 rather than IL-1 $\beta$ was identified as the critical inflammasome substrate driving NASH exacerbation because MCDD-fed IL-1 receptor knockout $\left(I l-1 r^{-/-}\right)$mice were not hypersusceptible to steatohepatitis, whereas Il-1 $18^{-/-}$mice were [12]. Moreover, cohousing $I l-18^{-/-}$mice with wild-type mice increased MCDD-induced NASH severity in the latter, further highlighting the transmissible nature of the phenotype. Together, these findings suggest that defective inflammasome signaling in the gastro-intestinal tract allows colitogenic microbes to prosper in the colon, and subsequently trigger harmful inflammatory signaling pathways in systemic organs when the gastro-intestinal barrier is breached (Figure 1). In this regard, microbial factors derived from the intestine were shown to trigger Tolllike receptor (TLR) activation in the liver after gaining access via the portal vein [14]. TLR signaling contributed to inflammasome-associated NASH exacerbation because mice deficient for the essential TLR adaptors MyD88 and TRIF failed to present with the expected increase in hepatic damage, inflammation and steatosis when cohoused with MCDD-fed $A S C^{-/-}$mice [12]. Similarly, co-housed $T l r 4^{-/-}$and $\mathrm{Tlr}^{9^{-/-}}$mice were protected from aggravated disease progression relative to singly-housed $\mathrm{Tlr}^{-/-}$and $\mathrm{Tlr} \mathrm{O}^{-/-}$ controls. Although live bacteria were not detected in the liver of MCDD-fed wild-type and $A S C^{-/-}$mice, increased levels of TLR4 and TLR9 agonists were present in the portal circulation of $A S C^{---}$and co-housed with wild-type mice compared to singly-housed wildtype mice [12]. High levels of CCL5 in the gut of inflammasome-deficient mice contributed to increased barrier disruption and TLR agonist translocation, as $\mathrm{CCL5}^{-1-}$ mice that were co-housed with $\mathrm{Nlrp}^{-/-}$or $A S \mathrm{C}^{-/-}$mice acquired the colitogenic species of inflammasomedeficient mice [6], but failed to develop severe MCDD-induced NASH [12], respectively. Concurrently, TLR agonist levels retrieved in the portal vein of $\mathrm{CCLS}^{-/-}$mice that were co-housed with $A S C^{-1-}$ mice were significantly lower than those of co-housed wildtype mice [12]. Thus, the increased CCL5 levels that are generated in response to the colitogenic intestinal milieu of inflammasome-deficient mice enhances translocation of microbial components to the liver, where excessive activation of TLR4 and TLR9 signaling contributes to enhanced NASH progression. In this regard, the TLR-induced cytokine TNF- $\alpha$ is known to mediate steatohepatitis and hepatic fibrosis in patients [15]. Concurrently, Tnf mRNA levels were found to be significantly upregulated in $\mathrm{Il}-18^{-/-}, \mathrm{ASC}^{-/-}$and co-housed wildtypemice that were subsequently fed MCDD, but not in MCDD-fed $\mathrm{Il}-1 \mathrm{r}^{-/-}$or singly-housed wild-type mice [12]. Furthermore, MCDD-fed $T n f^{-1-}$ mice that were co-housed with $A S C^{-1-}$ mice failed to develop the exacerbated disease seen in co-housed wildtype mice. In conclusion, inflammasome signaling in the gastro-intestinal tract is emerging as a remote control for metabolic disease manifestations in distant organs that keeps colitogenic microbes in check and prevents the damaging consequences of excessive translocation of inflammatory factors to systemic organs (Figure 1).

\section{Acknowledgments}

M Lamkanfi is supported by grants from European Union Marie-Curie (256432), ERC (281600) and the Fund for Scientific Research (G030212N, 1.2.201.10.N.00 and 1.5.122.11.N.00) Flanders. T-D K is supported by grants from NIAMS/NIH (R01AR056296) and the American Lebanese Syrian Associated Charities (ALSAC).

\section{References}

1 Qin J, Li R, Raes J, et al. A human gut microbial gene catalogue established by metagenomic sequencing. Nature 2010; 464:59-65.

2 Arumugam M, Raes J, Pelletier E, et $a l$. Enterotypes of the human gut microbiome. Nature 2011; 473:174-180.

3 Lamkanfi M, Walle LV, Kanneganti TD. Deregulated inflammasome signaling in disease. Immunol Rev 2011; 243:163-173.

4 Kanneganti TD, Lamkanfi M, Nunez G. Intracellular NOD-like receptors in host defense and disease. Immunity 2007; 27:549-559.

5 Lamkanfi M. Emerging inflammasome effector mechanisms. Nat Rev Immunol 2011; 11:213-220.

6 Elinav E, Strowig T, Kau AL, et al. NLRP6 inflammasome regulates colonic microbial ecology and risk for colitis. Cell 2011; 145:745-757.

7 Chen GY, Liu M, Wang F, Bertin J, Nunez G. A functional role for Nlrp6 in intestinal inflammation and tumorigenesis. J Immunol 2011; 186:7187-7194.

8 Normand S, Delanoye-Crespin A, Bressenot A, et al. Nod-like receptor pyrin domain-containing protein 6 (NLRP6) controls epithelial selfrenewal and colorectal carcinogenesis upon injury. Proc Natl Acad Sci USA 2011; 108:9601-9606.

9 Zaki MH, Boyd KL, Vogel P, et al. The NLRP3 inflammasome protects against loss of epithelial integrity and mortality during experimental colitis. Immunity 2010; 32:379-391.

10 Dupaul-Chicoine J, Yeretssian G, Doiron $\mathrm{K}$, et al. Control of intestinal homeostasis, colitis, and colitis-associated colorectal cancer by the inflammatory caspases. Immunity 2010; 32:367378.

11 Hirota SA, Ng J, Lueng A, et al. NLRP3 inflammasome plays a key role in the regulation of intestinal homeostasis. Inflamm Bowel Dis 2011; 17:1359-1372.

12 Henao-Mejia J, Elinav E, Jin C, et al. Inflammasome-mediated dysbiosis regulates progression of NAFLD and obesity. Nature 2012; 482:179-185.

13 Traggiai E, Chicha L, Mazzucchelli L, 
et al. Development of a human adaptive immune system in cord blood cell-transplanted mice. Science 2004; 304:104-107.

14 Seki E, Schnabl B. Role of innate im- munity and the microbiota in liver fibrosis: crosstalk between the liver and gut. J Physiol 2012; 590 (Pt 3):447458.

15 Li Z, Yang S, Lin H, et al. Probiotics and antibodies to TNF inhibit inflammatory activity and improve nonalcoholic fatty liver disease. Hepatology 2003; 37:343-350. 